\title{
Notes on Convergence Properties for a Smoothing-Regularization Approach to Mathematical Programs with Vanishing Constraints
}

\author{
Qingjie Hu, ${ }^{1}$ Yu Chen, ${ }^{1,2}$ Zhibin Zhu, ${ }^{1}$ and Bishan Zhang' \\ ${ }^{1}$ School of Mathematics and Computing Science, Guilin University of Electronic Technology, 541004 Guilin, China \\ ${ }^{2}$ School of Mathematics and Statistics, Central South University, 410083 Changsha, China \\ Correspondence should be addressed to Qingjie Hu; hqj0715@126.com
}

Received 24 June 2014; Accepted 29 July 2014; Published 1 September 2014

Academic Editor: Yunhai Xiao

Copyright (C) 2014 Qingjie Hu et al. This is an open access article distributed under the Creative Commons Attribution License, which permits unrestricted use, distribution, and reproduction in any medium, provided the original work is properly cited.

We give some improved convergence results about the smoothing-regularization approach to mathematical programs with vanishing constraints (MPVC for short), which is proposed in Achtziger et al. (2013). We show that the Mangasarian-Fromovitz constraints qualification for the smoothing-regularization problem still holds under the VC-MFCQ (see Definition 5) which is weaker than the VC-LICQ (see Definition 7) and the condition of asymptotic nondegeneracy. We also analyze the convergence behavior of the smoothing-regularization method and prove that any accumulation point of a sequence of stationary points for the smoothing-regularization problem is still strongly-stationary under the VC-MFCQ and the condition of asymptotic nondegeneracy.

\section{Introduction}

We consider the following mathematical program with vanishing constraints:

$$
\begin{array}{cl}
\min & f(z) \\
\text { s.t. } & g_{i}(z) \leq 0, \quad i=1,2, \ldots, m ; \\
& h_{j}(z)=0, \quad j=1,2, \ldots, p ; \\
& H_{i}(z) \geq 0, \quad i=1,2, \ldots, l \\
& G_{i}(z) H_{i}(z) \leq 0, \quad i=1,2, \ldots, l,
\end{array}
$$

where $f: R^{n} \rightarrow R, g: R^{n} \rightarrow R^{m}, h: R^{n} \rightarrow R^{p}$ and $G, H: R^{n} \rightarrow R^{l}$ are all continuously differentiable functions.

The MPVC was firstly introduced to the mathematical community in [1]. It plays an important role in some fields such as optimization topology design problems in mechanical structures [1] and robot path-finding problems with logic communication constraints in robot motion planning [2]. The major difficulty in solving problem (1) is that it does not satisfy some standard constraint qualifications at the feasible points so that the standard optimization methods are likely to fail for this problem. The MPVC has attracted much attention in the recent years. Several theoretical properties and different numerical approaches for MPVC can be found in [1-12]. Very recently, in [3], the authors have proposed a smoothing-regularization approach to mathematical programs with vanishing constraints. Their basic idea is to reformulate the characteristic constraints of the MPVC via a nonsmooth function and to eventually smooth it and regularize the feasible set with the aid of a certain smoothing and regularization parameter $\varepsilon>0$ such that the resulting problem is more tractable and coincides with the original program for $\varepsilon=0$. Under the VC-LICQ and the condition of asymptotic nondegeneracy, the convergence behaviors of a sequence of stationary points of the smoothing-regularized problems have been investigated.

In this note, we give some improved convergence results about the smoothing-regularization approach to mathematical programs with vanishing constraints in [3]. We show that these properties still hold under the weaker VC-MFCQ and the condition of asymptotic nondegeneracy. The smoothingregularization problems satisfy the standard MFCQ, which guarantees the existence of Lagrange multipliers at local minima; the sequence of multipliers is bounded, and the limit point is still strongly-stationary. 
The rest of the note is organized as follows. In Section 2, we review some concepts of the nonlinear programming and the MPVC and present the smoothing-regularization method for (1), which is proposed in [3]. In Section 3, we give the improved convergent properties. We close with some final remarks in Section 4.

For convenience of discussion, some notations to be used in this paper are given. The $i$ th component of $G$ will be denoted by $G_{i} ; X$ denotes the feasible set of problem (1). For a function $g: R^{n} \rightarrow R^{m}$ and a given vector $\alpha \in R^{n}$, we use $I_{g}(z)=\left\{i: g_{i}(z)=0\right\}$ and $\operatorname{supp}(\alpha)=\left\{i: \alpha_{i} \neq 0\right\}$ to denote the active index set of $g$ at $z$ and the support of $\alpha$, respectively.

\section{Preliminaries}

Firstly, we will introduce some definitions about the following optimization problem:

$$
\begin{array}{cl}
\min & f(z) \\
\text { s.t. } & g(z) \leq 0, \\
& h(z)=0,
\end{array}
$$

where $f: R^{n} \rightarrow R, g: R^{n} \rightarrow R^{m}, h: R^{n} \rightarrow R^{p}$ are all continuously differentiable functions. $F$ denotes the feasible set of problem (2).

Definition 1. A point $\bar{z} \in F$ is called a stationary point if there are multipliers $\lambda, \mu$ such that $(\bar{z}, \lambda, \mu)$ is a KKT point of (2); that is, the multipliers satisfy $\lambda \in R_{+}^{m}$ and $\mu \in R^{p}$ with $\lambda_{i} g_{i}(\bar{z})=0$ for all $i=1,2, \ldots, m$, and

$$
\nabla f(\bar{z})+\sum_{i=1}^{m} \lambda_{i} \nabla g_{i}(\bar{z})+\sum_{i=1}^{p} \mu_{i} \nabla h_{i}(\bar{z})=0 .
$$

Definition 2. A feasible point $\bar{z}$ of (2) is said to satisfy the Mangasarian-Fromovitz constraint qualification (MFCQ for short) if the gradients $\left\{\nabla h_{i}(\bar{z}) \mid i=1,2, \ldots, p\right\}$ are linearly independent and there is a $d \in R^{n}$ such that

$$
\begin{gathered}
\nabla g_{i}(\bar{z})^{T} d<0 \quad\left(i \in I_{g}(\bar{z})\right), \\
\nabla h_{i}(\bar{z})^{T} d=0 \quad(i=1,2, \ldots, p) .
\end{gathered}
$$

Definition 3 (see [13]). A finite set of vectors $\left\{a_{i} \mid i \in I_{1}\right\} \cup\left\{b_{i} \mid\right.$ $\left.i \in I_{2}\right\}$ is said to be positive-linearly dependent if there exists $(\alpha, \beta) \neq 0$ such that

$$
\sum_{i \in I_{1}} \alpha_{i} a_{i}+\sum_{i \in I_{2}} \beta_{i} b_{i}=0, \quad \alpha_{i} \geq 0, \quad \forall i \in I_{1} .
$$

If the above system only has a solution $(\alpha, \beta)=0$, we say that these vectors are positive-linearly independent.

By using Motzkin's theorem of the alternatives in [14], we can obtain the following property.

Lemma 4. A point $\bar{z} \in F$ satisfies the MFCQ if and only if the gradients

$$
\left\{\nabla g_{i}(\bar{z}) \mid i \in I_{g}(\bar{z})\right\} \cup\left\{\nabla h_{i}(\bar{z}) \mid i=1,2, \ldots, p\right\}
$$

are positive-linearly independent.
Now, we borrow notations from mathematical programs with complementarity constraints to define the following sets of active constraints in an arbitrary $\bar{z} \in X$ as follows:

$$
\begin{gathered}
I_{+}(\bar{z})=\left\{i \mid H_{i}(\bar{z})>0\right\}, \\
I_{0}(\bar{z})=\left\{i \mid H_{i}(\bar{z})=0\right\}, \\
I_{+0}(\bar{z})=\left\{i \mid H_{i}(\bar{z})>0, G_{i}(\bar{z})=0\right\}, \\
I_{+-}(\bar{z})=\left\{i \mid H_{i}(\bar{z})>0, G_{i}(\bar{z})<0\right\}, \\
I_{0+}(\bar{z})=\left\{i \mid H_{i}(\bar{z})=0, G_{i}(\bar{z})>0\right\}, \\
I_{00}(\bar{z})=\left\{i \mid H_{i}(\bar{z})=0, G_{i}(\bar{z})=0\right\}, \\
I_{0-}(\bar{z})=\left\{i \mid H_{i}(\bar{z})=0, G_{i}(\bar{z})<0\right\} .
\end{gathered}
$$

Definition 5 (see [1]). A feasible point $\bar{z}$ for (1) satisfies the vanishing constraints Mangasarian-Fromovitz constraints qualification (VC-MFCQ for short) if

$$
\begin{gathered}
\nabla h_{i}(\bar{z}) \quad(i=1,2, \ldots, p), \\
\nabla H_{i}(\bar{z}) \quad\left(i \in I_{0+}(\bar{z}) \cup I_{00}(\bar{z})\right)
\end{gathered}
$$

are linearly independent and there exists a vector $d \in R^{n}$ such that

$$
\begin{gathered}
\nabla h_{i}(\bar{z})^{T} d=0 \quad(i=1,2, \ldots, p), \\
\nabla H_{i}(\bar{z})^{T} d=0 \quad\left(i \in I_{0+}(\bar{z}) \cup I_{00}(\bar{z})\right), \\
\nabla g_{i}(\bar{z})^{T} d<0 \quad\left(i \in I_{g}(\bar{z})\right), \\
\nabla H_{i}(\bar{z})^{T} d>0 \quad\left(i \in I_{0-}(\bar{z})\right), \\
\nabla G_{i}(\bar{z})^{T} d<0 \quad\left(i \in I_{+0}(\bar{z})\right) .
\end{gathered}
$$

Similar to Lemma 4, we can also deduce the following result.

Lemma 6. A point $\bar{z} \in X$ satisfies the VC-MFCQ if and only if the gradients

$$
\begin{aligned}
\left\{\nabla g_{i}(\bar{z}) \mid i \in I_{g}(\bar{z})\right\} \\
\cup\left\{\nabla h_{i}(\bar{z}) \mid i=1,2, \ldots, p\right\} \\
\cup\left\{-\nabla H_{i}(\bar{z}) \mid i \in I_{0-}(\bar{z})\right\} \\
\cup\left\{\nabla G_{i}(\bar{z}) \mid i \in I_{+0}(\bar{z})\right\} \\
\quad \cup\left\{\nabla H_{i}(\bar{z}) \mid i \in I_{00}(\bar{z}) \cup I_{0+}(\bar{z})\right\}
\end{aligned}
$$

are positive-linearly independent. In other words, the MPVC at $\bar{z}$ satisfies the VC-MFCQ if and only if there does not exist a vector $\left(\lambda_{I_{g}(\bar{z})}, \mu, \alpha_{I_{0-}(\bar{z})}, \alpha_{I_{00}(\bar{z}) \cup I_{0+}(\bar{z})}, \beta_{I_{+0}(\bar{z})}\right) \neq 0$ with $\lambda_{i} \geq 0$ 
for all $i \in I_{g}(\bar{z}), \alpha_{i} \geq 0$ for all $i \in I_{0-}(\bar{z})$, and $\beta_{i} \geq 0$ for all $i \in I_{+0}(\bar{z})$ such that

$$
\begin{aligned}
\sum_{i \in I_{g}(\bar{z})} & \lambda_{i} \nabla g_{i}(\bar{z})+\sum_{i=1}^{l} \mu_{i} \nabla h_{i}(\bar{z}) \\
& -\sum_{i \in I_{0-}(\bar{z})} \alpha_{i} \nabla H_{i}(\bar{z})+\sum_{i \in I_{00}(\bar{z}) \cup I_{0+}(\bar{z})} \alpha_{i} \nabla H_{i}(\bar{z}) \\
& +\sum_{i \in I_{+0}(\bar{z})} \beta_{i} \nabla G_{i}(\bar{z})=0
\end{aligned}
$$

holds true.

Definition 7 (see [1]). A feasible point $\bar{z}$ for (1) satisfies the vanishing linear independence constraints qualification (VCLICQ for short) if and only if

$$
\begin{gathered}
\nabla h_{i}(\bar{z}) \quad(i=1,2, \ldots, p), \\
\nabla g_{i}(\bar{z}) \quad\left(i \in I_{g}(\bar{z})\right), \\
\nabla G_{i}(\bar{z}) \quad\left(i \in I_{+0}(\bar{z})\right), \\
\nabla H_{i}(\bar{z}) \quad\left(i \in I_{0}(\bar{z})\right)
\end{gathered}
$$

are linearly independent.

Remark 8. It is easy to see that the VC-LICQ implies the VCMFCQ. Moreover, the VC-LICQ (VC-MFCQ) is weaker than the MPVC-LICQ (MPVC-MFCQ) (See [7]).

Definition 9. Let $\bar{z}$ be a feasible point for the problem (1), then

(a) $\bar{z}$ is said to be weak-stationary if there exist multiplier vectors $\bar{\lambda} \in R^{m}, \bar{\mu} \in R^{p}$, and $\bar{u}, \bar{v} \in R^{l}$ such that

$$
\begin{gathered}
\nabla f(\bar{z})+\nabla g(\bar{z})^{T} \bar{\lambda}+\nabla h(\bar{z})^{T} \bar{\mu} \\
-\nabla H(\bar{z})^{T} \bar{v}+\nabla G(\bar{z})^{T} \bar{u}=0, \\
\bar{\lambda} \geq 0, \quad \bar{z} \in X, \quad \bar{\lambda}^{T} g(\bar{z})=0, \\
\bar{v}_{i}=0 \quad\left(i \in I_{+}(\bar{z})\right), \\
\bar{v}_{i} \geq 0 \quad\left(i \in I_{0-}(\bar{z})\right), \\
\bar{v}_{i} \quad \text { free } \quad\left(i \in I_{0+}(\bar{z}) \cup I_{00}(\bar{z})\right), \\
\bar{u}_{i}=0 \quad\left(i \in I_{+-}(\bar{z}) \cup I_{0-}(\bar{z}) \cup I_{0+}(\bar{z})\right), \\
\bar{u}_{i} \geq 0 \quad\left(i \in I_{+0}(\bar{z}) \cup I_{00}(\bar{z})\right) .
\end{gathered}
$$

(b) $\bar{z}$ is said to be strongly-stationary, if it is weakstationary and

$$
\bar{u}_{i}=0, \quad \bar{v}_{i} \geq 0, \quad i \in I_{00}(\bar{z}) .
$$

Finally, we give the smoothing-regularization method of Problem (1), which is proposed in [3]. According to [3], with the help of a positive parameter, the MPVC (1) is approximated by the following smoothing-regularization problem:

$$
\begin{array}{cl}
\min & f(z) \\
\text { s.t. } & g(z) \leq 0, \quad h(z)=0, \\
& r_{\varepsilon}(z) \leq \varepsilon,
\end{array}
$$

where

$$
\begin{gathered}
r_{\varepsilon}(z)=\left(\begin{array}{c}
r_{\varepsilon, 1}(z) \\
\vdots \\
r_{\varepsilon, l}(z)
\end{array}\right), \\
r_{\varepsilon, i}(z)=\frac{1}{2}\left(G_{i}(z) H_{i}(z)+\sqrt{\left(G_{i}(z) H_{i}(z)\right)^{2}+\varepsilon^{2}}\right. \\
\left.+\sqrt{\left(H_{i}(z)\right)^{2}+\varepsilon^{2}}-H_{i}(z)\right) .
\end{gathered}
$$

In order to give our improved convergence analysis, the following concept of asymptotic nondegeneracy is necessary.

Definition 10 (see [3]). Let $\bar{z}$ be feasible for the MPVC (1). Then a sequence $\left\{z_{k}\right\}$ of feasible points for (15) converging to $\bar{z}$ as $\varepsilon_{k} \downarrow 0$ is called asymptotically nondegenerate, if any accumulation point of $\left\{\nabla r_{\varepsilon_{k}, i}\left(z_{k}\right)\right\}$ is different from 0 for each $i \in I_{+0}(\bar{z}) \cup I_{0}(\bar{z})$.

\section{Some Improved Convergence Properties}

In this section, we will consider the improved convergence properties of a sequence of stationary points for the smoothing-regularization problem (15). Firstly, we discuss the constraint qualification of (15).

For convenience of discussion, we give the following notations:

$$
\begin{gathered}
a_{\varepsilon, i}(z)=H_{i}(z)+\frac{G_{i}(z) H_{i}(z)^{2}}{\sqrt{G_{i}(z)^{2} H_{i}(z)^{2}+\varepsilon^{2}}}, \\
b_{\varepsilon, i}(z)=G_{i}(z)+\frac{G_{i}(z)^{2} H_{i}(z)}{\sqrt{G_{i}(z)^{2} H_{i}(z)^{2}+\varepsilon^{2}}}+\frac{H_{i}(z)}{\sqrt{H_{i}(z)^{2}+\varepsilon^{2}}}-1, \\
I_{r_{\varepsilon}}(z)=\left\{i: r_{\varepsilon, i}(z)=\varepsilon\right\} .
\end{gathered}
$$

To show that the Mangasarian-Fromovitz constraints qualification for the problem (15) holds under some conditions, the following lemma palys a very important role.

Lemma 11. Let $\bar{z}$ be feasible for (1) such that the VC-MFCQ is satisfied at $\bar{z}$ and the sequence $\left\{z_{k}\right\}$ of feasible points for (15) 
converging to $\bar{z}$ as $\varepsilon_{k} \downarrow 0$ is asymptotically nondegenerate. Then, for sufficiently large $k$, the set of vectors

$$
\begin{gathered}
\nabla g_{i}\left(z_{k}\right), \quad i \in I_{g}(\bar{z}), \\
\nabla h_{i}\left(z_{k}\right), \quad i=1,2, \ldots, p, \\
-\left(a_{\varepsilon_{k}, i}\left(z_{k}\right) \nabla G_{i}\left(z_{k}\right)+b_{\varepsilon_{k}, i}\left(z_{k}\right) \nabla H_{i}\left(z_{k}\right)\right), \\
i \in I_{r_{\varepsilon_{k}}}\left(z_{k}\right) \cap I_{0-}(\bar{z}), \\
a_{\varepsilon_{k}, i}\left(z_{k}\right) \nabla G_{i}\left(z_{k}\right)+b_{\varepsilon_{k}, i}\left(z_{k}\right) \nabla H_{i}\left(z_{k}\right), \\
i \in I_{\varepsilon_{\varepsilon_{k}}}\left(z_{k}\right) \cap I_{+0}(\bar{z}), \\
a_{\varepsilon_{\varepsilon_{k}}, i}\left(z_{k}\right) \nabla G_{i}\left(z_{k}\right)+b_{\varepsilon_{k}, i}\left(z_{k}\right) \nabla H_{i}\left(z_{k}\right), \\
i \in I_{r_{\varepsilon_{k}}}\left(z_{k}\right) \cap\left(I_{0+}(\bar{z}) \cup I_{00}(\bar{z})\right)
\end{gathered}
$$

are positive-linearly independent.

Proof. Since $g, h, G, H$ are all continuous, for sufficiently large $k$, we have

$$
I_{g}\left(z_{k}\right) \subseteq I_{g}(\bar{z}), \quad I_{h}\left(z_{k}\right) \subseteq I_{h}(\bar{z}) .
$$

Because the VC-MFCQ holds, the gradients

$$
\begin{aligned}
\left\{\nabla g_{i}(\bar{z}) \mid i \in I_{g}(\bar{z})\right\} \\
\cup\left\{\nabla h_{i}(\bar{z}) \mid i=1,2, \ldots, p\right\} \\
\cup\left\{-\nabla H_{i}(\bar{z}) \mid i \in I_{0-}(\bar{z})\right\} \\
\cup\left\{\nabla G_{i}(\bar{z}) \mid i \in I_{+0}(\bar{z})\right\} \\
\quad \cup\left\{\nabla H_{i}(\bar{z}) \mid i \in I_{00}(\bar{z}) \cup I_{0+}(\bar{z})\right\}
\end{aligned}
$$

are positive-linearly independent by Lemma 6 , taking into account that

$$
\begin{gathered}
\left(I_{r_{\varepsilon_{k}}}\left(z_{k}\right) \cap I_{0-}(\bar{z})\right) \subseteq I_{0-}(\bar{z}), \\
\left(I_{r_{\varepsilon_{k}}}\left(z_{k}\right) \cap I_{+0}(\bar{z})\right) \subseteq I_{+0}(\bar{z}), \\
\left(I_{r_{\varepsilon_{k}}}\left(z_{k}\right) \cap I_{0+}(\bar{z})\right) \cup\left(I_{r_{\varepsilon_{k}}}\left(z_{k}\right) \cap I_{00}(\bar{z})\right) \\
\subseteq I_{00}(\bar{z}) \cup I_{0+}(\bar{z}) .
\end{gathered}
$$

In view of the condition of asymptotic nondegeneracy, we know that $a_{\varepsilon_{k}, i}\left(z_{k}\right) \neq 0, b_{\varepsilon_{k}, i}\left(z_{k}\right) \approx 0$ for all $i \in I_{+0}(\bar{z})$ and $a_{\varepsilon_{k}, i}\left(z_{k}\right) \approx 0, b_{\varepsilon_{k}, i}\left(z_{k}\right) \neq 0$ for $i \in I_{0}$ for all sufficiently large $k$.
Similar to the proof of Proposition 2.2 in [15], we know that the set of vectors

$$
\begin{gathered}
\nabla g_{i}\left(z_{k}\right), \quad i \in I_{g}(\bar{z}), \\
\nabla h_{i}\left(z_{k}\right), \quad i=1,2, \ldots, p, \\
-\left(a_{\varepsilon_{k}, i}\left(z_{k}\right) \nabla G_{i}\left(z_{k}\right)+b_{\varepsilon_{k}, i}\left(z_{k}\right) \nabla H_{i}\left(z_{k}\right)\right), \\
i \in I_{r_{\varepsilon_{k}}}\left(z_{k}\right) \cap I_{0-}(\bar{z}), \\
a_{\varepsilon_{\varepsilon_{k}}, i}\left(z_{k}\right) \nabla G_{i}\left(z_{k}\right)+b_{\varepsilon_{k}, i}\left(z_{k}\right) \nabla H_{i}\left(z_{k}\right), \\
i \in I_{\varepsilon_{\varepsilon_{k}}}\left(z_{k}\right) \cap I_{+0}(\bar{z}), \\
a_{\varepsilon_{k_{k}}, i}\left(z_{k}\right) \nabla G_{i}\left(z_{k}\right)+b_{\varepsilon_{k}, i}\left(z_{k}\right) \nabla H_{i}\left(z_{k}\right), \\
i \in I_{r_{\varepsilon_{k}}}\left(z_{k}\right) \cap\left(I_{0+}(\bar{z}) \cup I_{00}(\bar{z})\right)
\end{gathered}
$$

are positive-linearly independent for all sufficiently large $k$. The proof is completed.

Based on the above lemma, we can show the following theorem.

Theorem 12. Let $\bar{z}$ be feasible for (1) such that the VC-MFCQ is satisfied at $\bar{z}$ and the sequence $\left\{z_{k}\right\}$ of feasible points for (15) converging to $\bar{z}$ as $\varepsilon_{k} \downarrow 0$ is asymptotically nondegenerate. Then, for sufficiently large $k$, Problem (15) satisfies the standard MFCQ at $z_{k}$.

Proof. Taking Lemma 11 into account, we know that the set of vectors

$$
\begin{gathered}
\nabla g_{i}\left(z_{k}\right), \quad i \in I_{g}(\bar{z}), \\
\nabla h_{i}\left(z_{k}\right), \quad i=1,2, \ldots, p, \\
-\left(a_{\varepsilon_{k}, i}\left(z_{k}\right) \nabla G_{i}\left(z_{k}\right)+b_{\varepsilon_{k}, i}\left(z_{k}\right) \nabla H_{i}\left(z_{k}\right)\right), \\
i \in I_{r_{\varepsilon_{k}}}\left(z_{k}\right) \cap I_{0-}(\bar{z}), \\
a_{\varepsilon_{k_{k}}, i}\left(z_{k}\right) \nabla G_{i}\left(z_{k}\right)+b_{\varepsilon_{k}, i}\left(z_{k}\right) \nabla H_{i}\left(z_{k}\right), \\
i \in I_{\varepsilon_{\varepsilon_{k}}}\left(z_{k}\right) \cap I_{+0}(\bar{z}), \\
a_{\varepsilon_{\varepsilon_{k}}, i}\left(z_{k}\right) \nabla G_{i}\left(z_{k}\right)+b_{\varepsilon_{k}, i}\left(z_{k}\right) \nabla H_{i}\left(z_{k}\right), \\
i \in I_{r_{\varepsilon_{k}}}\left(z_{k}\right) \cap\left(I_{0+}(\bar{z}) \cup I_{00}(\bar{z})\right)
\end{gathered}
$$

are positive-linearly independent for sufficiently large $k$.

We now prove that the standard MFCQ holds at $z_{k}$ for Problem (15) for sufficiently large $k$. In view of Lemma 4 , we have to show that

$$
\begin{aligned}
0= & \sum_{i \in I_{g}\left(z_{k}\right)} \lambda_{i}^{k} \nabla g_{i}\left(z_{k}\right)+\sum_{i=1}^{p} \mu_{i}^{k} \nabla h_{i}\left(z_{k}\right) \\
& +\sum_{i=1}^{l} \gamma_{i}^{k}\left(a_{\varepsilon_{k}, i}\left(z_{k}\right) \nabla G_{i}\left(z_{k}\right)+b_{\varepsilon_{k}, i}\left(z_{k}\right) \nabla H_{i}\left(z_{k}\right)\right)
\end{aligned}
$$


with $\mu^{k} \in R^{p}$ and $\lambda^{k}, \gamma^{k} \geq 0$ holds for the zero vector. To see this, we rewrite (24) as

$$
\begin{aligned}
& 0=\sum_{i \in I_{g}\left(z_{k}\right)} \lambda_{i}^{k} \nabla g_{i}\left(z_{k}\right)+\sum_{i=1}^{p} \mu_{i}^{k} \nabla h_{i}\left(z_{k}\right) \\
& -\sum_{i \in I_{I_{\varepsilon_{k}}}\left(z_{k}\right) \cap I_{0-}(\bar{z})} \gamma_{i}^{k}\left(-a_{\varepsilon_{k}, i}\left(z_{k}\right) \nabla G_{i}\left(z_{k}\right)\right. \\
& \left.-b_{\varepsilon_{k}, i}\left(z_{k}\right) \nabla H_{i}\left(z_{k}\right)\right) \\
& +\sum_{i \in I_{r_{\varepsilon_{k}}}\left(z_{k}\right) \cap I_{+0}(z)} \gamma_{i}^{k}\left(a_{\varepsilon_{k}, i}\left(z_{k}\right) \nabla G_{i}\left(z_{k}\right)\right. \\
& \left.+b_{\varepsilon_{k}, i}\left(z_{k}\right) \nabla H_{i}\left(z_{k}\right)\right) \\
& +\sum_{i \in I_{I_{\varepsilon_{k}}}\left(z_{k}\right) \cap\left(I_{0+}(\bar{z}) \cup I_{00}(\bar{z})\right)} \gamma_{i}^{k}\left(a_{\varepsilon_{k}, i}\left(z_{k}\right) \nabla G_{i}\left(z_{k}\right)\right. \\
& \left.+b_{\varepsilon_{k}, i}\left(z_{k}\right) \nabla H_{i}\left(z_{k}\right)\right) \text {. }
\end{aligned}
$$

In view of the condition of asymptotic nondegeneracy, applying the positive linear independence of vectors from (23) to (25) and (19), one gets

$$
\begin{gathered}
\lambda_{i}^{k}=0 \quad\left(i \in I_{g}\left(z_{k}\right)\right), \\
\mu_{i}^{k}=0 \quad(i=1,2, \ldots, p), \\
\gamma_{i}^{k}=0 \quad\left(i \in I_{r_{\varepsilon_{k}}}\left(z^{k}\right) \cap I_{0-}(\bar{z})\right), \\
\gamma_{i}^{k}=0 \quad\left(i \in I_{r_{\varepsilon_{k}}}\left(z_{k}\right) \cap I_{+0}(\bar{z})\right), \\
\gamma_{i}^{k}=0 \quad\left(i \in I_{r_{\varepsilon_{k}}}\left(z^{k}\right) \cap\left(I_{0+}(\bar{z}) \cup I_{00}(\bar{z})\right)\right) .
\end{gathered}
$$

The proof is completed.

Remark 13. In Theorem 12, by relaxing the condition of the VC-LICQ, we show that the VC-MFCQ and the condition of asymptotic nondegeneracy imply that the smoothingregularization problems satisfy the standard MFCQ. Hence, Theorem 12 is an improved version of Lemma 5.6 in [3].

To establish the relations between the solutions of the original problem and those of the smoothing-regularization problem under the VC-MFCQ and the condition of asymptotic nondegeneracy, we give the following key lemma.

Lemma 14. Let $\varepsilon_{k}>0$ be convergent to zero. Suppose that $\left\{z_{k}\right\}$ is a sequence of stationary points of Problem (15) with $\varepsilon=\varepsilon_{k}$ and $\left(\lambda^{k}, \mu^{k}, \gamma^{k}\right)$ being the corresponding multiplier vectors. If $\bar{z}$ is an accumulation point of the sequence $\left\{z_{k}\right\}$ such that the VC-MFCQ holds at $\bar{z}$ and the condition of asymptotic nondegeneracy for $\left\{z_{k}\right\}$ is satisfied, then the sequence of multipliers $\left\{\left(\lambda^{k}, \mu^{k}, \gamma^{k}\right)\right\}$ is bounded.
Proof. It follows from Theorem 12 that, for sufficiently large $k$, there exist lagrangian multiplier vectors $\left(\lambda^{k}, \mu^{k}, \gamma^{k}\right)$ such that

$$
\begin{gathered}
\nabla f\left(z_{k}\right)+\sum_{i=1}^{m} \lambda_{i}^{k} \nabla g_{i}\left(z_{k}\right)+\sum_{i=1}^{p} \mu_{i}^{k} \nabla h_{i}\left(z_{k}\right) \\
+\sum_{i=1}^{l} \gamma_{i}^{k} \nabla r_{\varepsilon_{k}, i}\left(z_{k}\right)=0, \\
\lambda_{k} \geq 0, \quad \operatorname{supp}\left(\lambda^{k}\right) \subseteq I_{g}\left(z_{k}\right), \\
\gamma_{k} \geq 0, \quad \operatorname{supp}\left(\gamma^{k}\right) \subseteq I_{r_{\varepsilon_{k}}}\left(z_{k}\right) .
\end{gathered}
$$

From (27), we have

$$
\begin{aligned}
\nabla f\left(z_{k}\right) & +\sum_{i \in \operatorname{supp}\left(\lambda_{k}\right)} \lambda_{i}^{k} \nabla g_{i}\left(z_{k}\right) \\
& +\sum_{i \in \operatorname{supp}\left(\mu_{k}\right)} \mu_{i}^{k} \nabla h_{i}\left(z_{k}\right) \\
& +\sum_{i \in \operatorname{supp}\left(\gamma_{k}\right) \cap I_{0-}(\bar{z})} \gamma_{i}^{k} a_{\varepsilon_{k}, i}\left(z_{k}\right) \nabla G_{i}\left(z_{k}\right) \\
& +\sum_{i \in \operatorname{supp}\left(\gamma_{k}\right) \cap I_{0-}(\bar{z})} \gamma_{i}^{k} b_{\varepsilon_{k}, i}\left(z_{k}\right) \nabla H_{i}\left(z_{k}\right) \\
& +\sum_{i \in \operatorname{supp}\left(\gamma_{k}\right) \cap I_{+0}(\bar{z})} \gamma_{i}^{k} a_{\varepsilon_{k}, i}\left(z_{k}\right) \nabla G_{i}\left(z_{k}\right) \\
& +\sum_{i \in \operatorname{supp}\left(\gamma_{k}\right) \cap I_{+0}(\bar{z})} \gamma_{i}^{k} b_{\varepsilon_{k}, i}\left(z_{k}\right) \nabla H_{i}\left(z_{k}\right) \\
& +\sum_{i \in \operatorname{supp}\left(\gamma_{k}\right) \cap\left(I_{0^{+}}(\bar{z}) \cup I_{00}(\bar{z})\right)} \gamma_{i}^{k} a_{\varepsilon_{k}, i}\left(z_{k}\right) \nabla G_{i}\left(z_{k}\right) \\
& +\sum_{i \in \operatorname{supp}\left(\gamma_{k}\right) \cap\left(I_{0+}(\bar{z}) \cup I_{00}(\bar{z})\right)} \gamma_{i}^{k} b_{\varepsilon_{k}, i}\left(z_{k}\right) \nabla H_{i}\left(z_{k}\right)=0 .
\end{aligned}
$$

We can define

$$
\begin{gathered}
\beta_{i}^{k}= \begin{cases}-\gamma_{i}^{k} b_{\varepsilon_{k}, i}\left(z_{k}\right), & i \in \operatorname{supp}\left(\gamma^{k}\right) \cap I_{0-}(\bar{z}) ; \\
0, & \text { otherwise, }\end{cases} \\
\bar{\gamma}_{i}^{k}= \begin{cases}\gamma_{i}^{k} a_{\varepsilon_{k}, i}\left(z_{k}\right), & i \in \operatorname{supp}\left(\gamma^{k}\right) \cap I_{+0}(\bar{z}) ; \\
0, & \text { otherwise, }\end{cases} \\
\bar{\nu}_{i}^{k}= \begin{cases}\gamma_{i}^{k} b_{\varepsilon_{k}, i}\left(z_{k}\right), & i \in \operatorname{supp}\left(\gamma^{k}\right) \cap\left(I_{0+}(\bar{z}) \cup I_{00}(\bar{z})\right) ; \\
0, & \text { otherwise. }\end{cases}
\end{gathered}
$$


Noting that with $\beta_{i}^{k}, \bar{\gamma}_{i}^{k}$, and $\bar{v}_{i}^{k}$, (27) can be rewritten as

$$
\begin{aligned}
0= & \nabla f\left(z_{k}\right)+\sum_{i=1}^{m} \lambda_{i}^{k} \nabla g_{i}\left(z_{k}\right) \\
& +\sum_{i=1}^{p} \mu_{i}^{k} \nabla h_{i}\left(z_{k}\right)+\sum_{i=1}^{l} \beta_{i}^{k}\left(-\nabla H_{i}\left(z_{k}\right)\right) \\
& +\sum_{i=1}^{l} \bar{\gamma}_{i}^{k} \nabla G_{i}\left(z_{k}\right)+\sum_{i=1}^{l} \bar{\nu}_{i}^{k} \nabla H_{i}\left(z_{k}\right) \\
& +\sum_{i \in \operatorname{supp}\left(\gamma_{k}\right) \cap\left(I_{0+}(\bar{z}) \cup I_{00}(\bar{z})\right)} \gamma_{i}^{k} a_{\varepsilon_{k}, i}\left(z_{k}\right) \nabla G_{i}\left(z_{k}\right) \\
& +\sum_{i \in \operatorname{supp}\left(\gamma_{k}\right) \cap I_{+0}(\bar{z})} \gamma_{i}^{k} b_{\varepsilon_{k}, i}\left(z_{k}\right) \nabla H_{i}\left(z_{k}\right) \\
& +\sum_{i \in \operatorname{supp}\left(\gamma_{k}\right) \cap I_{0-}(\bar{z})} \gamma_{i}^{k} a_{\varepsilon_{k}, i}\left(z_{k}\right) \nabla G_{i}\left(z_{k}\right) .
\end{aligned}
$$

The following objective is to prove that the sequence $\left\{\left(\lambda^{k}, \mu^{k}, \beta^{k}, \bar{\gamma}^{k}, \bar{\nu}^{k}, \gamma_{I_{+0}(\bar{z}) \cup I_{0+}(\bar{z}) \cup I_{00}(\bar{z}) \cup I_{0-}(\bar{z})}^{k}\right)\right\}$ is bounded.

Assume that the sequence $\left\{\left(\lambda^{k}, \mu^{k}, \beta^{k}, \bar{\gamma}^{k}, \bar{\nu}^{k}\right.\right.$, $\left.\left.\gamma_{I_{+0}(\bar{z}) \cup I_{0+}(\bar{z}) \cup I_{00}(\bar{z}) \cup I_{0-}(\bar{z})}^{k}\right)\right\}$ is unbounded. Then, there exists a subset $K$ such that

$$
\begin{aligned}
& \left\|\left(\lambda^{k}, \mu^{k}, \beta^{k}, \bar{\gamma}^{k}, \bar{\nu}^{k}, \gamma_{I_{+0}(\bar{z}) \cup I_{0+}(\bar{z}) \cup I_{00}(\bar{z}) \cup I_{0-}(\bar{z})}^{k}\right)_{K}\right\| \\
& \longrightarrow+\infty \quad(k \longrightarrow+\infty) .
\end{aligned}
$$

So the corresponding normed sequence converges:

$$
\begin{aligned}
& \frac{\left(\lambda^{k}, \mu^{k}, \beta^{k}, \bar{\gamma}^{k}, \bar{\nu}^{k}, \gamma_{I_{+0}(\bar{z}) \cup I_{0+}(\bar{z}) \cup I_{00}(\bar{z}) \cup I_{0-}(\bar{z})}^{k}\right)}{\left\|\left(\lambda^{k}, \mu^{k}, \beta^{k}, \bar{\gamma}^{k}, \bar{\nu}^{k}, \gamma_{I_{+0}(\bar{z}) \cup I_{0+}(\bar{z}) \cup I_{00}(\bar{z}) \cup I_{0-}(\bar{z})}^{k}\right)\right\|} \\
& \underset{k \in K}{\longrightarrow}\left(\lambda, \mu, \beta, \bar{\gamma}, \bar{\nu}, \gamma_{I_{+0}(\bar{z}) \cup I_{0+}(\bar{z}) \cup I_{00}(\bar{z}) \cup I_{0-}(\bar{z})}\right) \neq 0 .
\end{aligned}
$$

Combined with (31), it yields

$$
\begin{aligned}
0= & \sum_{i=1}^{m} \lambda_{i} \nabla g_{i}(\bar{z})+\sum_{i=1}^{p} \mu_{i} \nabla h_{i}(\bar{z}) \\
& +\sum_{i=1}^{l} \beta_{i}\left(-\nabla H_{i}(\bar{z})\right)+\sum_{i=1}^{l} \bar{\gamma}_{i} \nabla G_{i}(\bar{z})+\sum_{i=1}^{l} \bar{\nu}_{i} \nabla H_{i}(\bar{z}),
\end{aligned}
$$

that is,

$$
\begin{aligned}
0= & \sum_{i \in \operatorname{supp}(\lambda)} \lambda_{i} \nabla g_{\mathrm{i}}(\bar{z})+\sum_{i \in \operatorname{supp}(\mu)} \mu_{i} \nabla h_{i}(\bar{z}) \\
& +\sum_{i \in \operatorname{supp}(\beta)} \beta_{i}\left(-\nabla H_{i}(\bar{z})\right)+\sum_{i \in \operatorname{supp}(\bar{\gamma})} \bar{\gamma}_{i} \nabla G_{i}(\bar{z}) \\
& +\sum_{i \in \operatorname{supp}(\bar{v})} \bar{\nu}_{i} \nabla H_{i}(\bar{z})
\end{aligned}
$$

where $\lambda \geq 0$ and, for all $k \in K$ being large enough,

$$
\begin{aligned}
& \operatorname{supp}(\lambda) \subseteq I_{g}\left(z_{k}\right) \subseteq I_{g}(\bar{z}) \\
& \operatorname{supp}(\beta) \subseteq \operatorname{supp}\left(\beta^{k}\right) \subseteq\left(\operatorname{supp}\left(\gamma^{k}\right) \cap I_{0-}(\bar{z})\right) \subseteq I_{0-}(\bar{z}), \\
& \operatorname{supp}(\bar{\gamma}) \subseteq \operatorname{supp}\left(\bar{\gamma}^{k}\right) \\
& \subseteq\left(\operatorname{supp}\left(\gamma^{k}\right) \cap I_{+0}(\bar{z})\right) \subseteq I_{+0}(\bar{z}), \\
& \operatorname{supp}(\bar{\gamma}) \subseteq \operatorname{supp}\left(\bar{\gamma}^{k}\right) \\
& \subseteq\left(\operatorname{supp}\left(\gamma^{k}\right) \cap\left(I_{00}(\bar{z}) \cup I_{0+}(\bar{z})\right)\right) \\
& \subseteq I_{00}(\bar{z}) \cup I_{0+}(\bar{z}) .
\end{aligned}
$$

We can prove that $(\lambda, \mu, \beta, \bar{\gamma}, \bar{\nu}) \neq 0$. Actually, if $(\lambda, \mu$, $\beta, \bar{\gamma}, \bar{\nu})=0$, then, for at least one $i \in I_{+0}(\bar{z}) \cup I_{0+}(\bar{z}) \cup$ $I_{00}(\bar{z}) \cup I_{0-}(\bar{z}), \gamma_{i} \neq 0$. Without loss of generality, assume that $\gamma_{i} \neq 0$ for an $i \in I_{+0}(\bar{z})$, then, for all $k$ sufficiently large, $\gamma_{i}^{k} \neq 0$. Consequently, $\bar{\gamma}_{i}^{k}=\gamma_{i}^{k} a_{\varepsilon_{k}, i}\left(z_{k}\right)$ for those $k$. Taking into account the condition of asymptotic nondegeneracy, for $i \in I_{+0}(\bar{z})$, we have

$$
\bar{\gamma}_{i}=\lim _{k \in K} \bar{\gamma}_{i}^{k}=\lim _{k \in K} \gamma_{i}^{k} a_{\varepsilon_{k}, i}\left(z_{k}\right) \neq 0,
$$

which contradicts the assumption $\bar{\gamma}=0$.

By Lemma 6 , we know that $(\lambda, \mu, \beta, \bar{\gamma}, \bar{\nu}) \neq 0$ contradicts the fact that the VC-MFCQ holds at $\bar{z}$. Thus, the sequence $\left\{\left(\lambda^{k}, \mu^{k}, \beta^{k}, \bar{\gamma}^{k}, \bar{\nu}^{k}, \gamma_{I_{+0}}^{k}(\bar{z}) \cup I_{0+}(\bar{z}) \cup I_{00}(\bar{z}) \cup I_{0-}(\bar{z})\right)\right\}$ is bounded.

Again, noting the condition of asymptotic nondegeneracy and the definitions of $\beta_{i}^{k}, \bar{\gamma}_{i}^{k}, \bar{v}_{i}^{k}$, we can prove that the sequence of multipliers $\left\{\left(\lambda^{k}, \mu^{k}, \gamma^{k}\right)\right\}$ are bounded. The proof is completed.

Based on Lemma 14, similar to the proof of Theorem 5.3 in [3], we can obtain the following convergence result.

Theorem 15. Let $\varepsilon_{k}>0$ be convergent to zero. Suppose that $\left\{z_{k}\right\}$ is a sequence of stationary points of Problem (15) with $\varepsilon=\varepsilon_{k}$. If $\bar{z}$ is an accumulation point of the sequence $\left\{z_{k}\right\}$ such that the VC-MFCQ holds at $\bar{z}$ and the condition of asymptotic nondegeneracy for $\left\{z_{k}\right\}$ is satisfied, then $\bar{z}$ is a strongly-stationary point of Problem (1).

Remark 16. In Theorem 15, by replacing the condition of the VC-LICQ, we prove that any accumulation point of stationary points for the smoothing-regularization problem is still strongly-stationary under the VC-MFCQ and the condition of asymptotic nondegeneracy. Hence, Theorem 15 includes Theorem 5.3 in [3] as a special case.

\section{Concluding Remarks}

In this note, we have shown that the VC-LICQ assumption can be replaced by the weaker VC-MFCQ condition in order to get the strong stationarity for the smoothing-regularization 
approach to mathematical programs with vanishing constraints, which is proposed in [3]. We have also shown that the VC-MFCQ implies that the smoothing-regularization problems satisfy the standard MFCQ. While it seems possible to prove that many other VC-tailored constraint qualifications imply that the corresponding standard constraint qualification holds for the smoothing-regularization problem, it is an open question whether one can further relax the VC-MFCQ assumption to get strong stationarity in the limit.

\section{Conflict of Interests}

The authors declare that there is no conflict of interests regarding the publication of this paper.

\section{Acknowledgments}

This work is supported by NNSF (nos. 11371073, 11461015, 11361018) of China, Guangxi Natural Science Foundation (no. 2014GXNSFFA118001), and Guangxi Fund for Distinguished Young Scholars (no. 2012GXSFFA060003).

\section{References}

[1] W. Achtziger and C. Kanzow, "Mathematical programs with vanishing constraints: optimality conditions and constraint qualifications," Mathematical Programming, vol. 114, no. 1, pp. 69-99, 2008.

[2] C. Kirches, A. Potschka, H. G. Bock, and S. Sager, "A parametric active set method for quadratic programs with vanishing constraints," Technical Report, Interdisciplinary Center for Scientic Computing, University of Heidelberg, Heidelberg, Germany, 2012.

[3] W. Achtziger, T. Hoheisel, and C. Kanzow, "A smoothingregularization approach to mathematical programs with vanishing constraints," Computational Optimization and Applications, vol. 55, no. 3, pp. 733-767, 2013.

[4] W. Achtziger, C. Kanzow, and T. Hoheisel, "On a relaxation method for mathematical programs with vanishing constraints," GAMM-Mitteilungen, vol. 35, no. 2, pp. 110-130, 2012.

[5] D. Dorsch, V. Shikhman, and O. Stein, "Mathematical programs with vanishing constraints: critical point theory," Journal of Global Optimization, vol. 52, no. 3, pp. 591-605, 2012.

[6] T. Hoheisel and C. Kanzow, "On the Abadie and Guignard constraint qualifications for mathematical programmes with vanishing constraints," Optimization, vol. 58, no. 4, pp. 431-448, 2009.

[7] T. Hoheisel and C. Kanzow, "Stationary conditions for mathematical programs with vanishing constraints using weak constraint qualifications," Journal of Mathematical Analysis and Applications, vol. 337, no. 1, pp. 292-310, 2008.

[8] T. Hoheisel and C. Kanzow, "First- and second-order optimality conditions for mathematical programs with vanishing constraints," Applications of Mathematics, vol. 52, no. 6, pp. 495514, 2007.

[9] T. Hoheisel, C. Kanzow, and J. V. Outrata, "Exact penalty results for mathematical programs with vanishing constraints," Nonlinear Analysis: Theory, Methods \& Applications, vol. 72, no. 5, pp. 2514-2526, 2010.
[10] T. Hoheisel, C. Kanzow, and A. Schwartz, "Convergence of a local regularization approach for mathematical programmes with complementarity or vanishing constraints," Optimization Methods \& Software, vol. 27, no. 3, pp. 483-512, 2012.

[11] A. F. Izmailov and A. L. Pogosyan, "Optimality conditions and Newton-type methods for mathematical programs with vanishing constraints," Computational Mathematics and Mathematical Physics, vol. 49, no. 7, pp. 1128-1140, 2009.

[12] A. F. Izmailov and M. V. Solodov, "Mathematical programs with vanishing constraints: optimality conditions, sensitivity, and a relaxation method," Journal of Optimization Theory and Applications, vol. 142, no. 3, pp. 501-532, 2009.

[13] C. Davis, "Theory of positive linear dependence," The American Journal of Mathematics, vol. 76, no. 4, pp. 733-746, 1954.

[14] O. L. Mangasarian, Nonliear Programming, McGraw-Hill, New York, NY, USA, 1969, reprinted by SIAM, Philadelphia, Pa, USA, 1994.

[15] L. Qi and Z. Wei, "On the constant positive linear dependence condition and its application to SQP methods," SIAM Journal on Optimization, vol. 10, no. 4, pp. 963-981, 2000. 


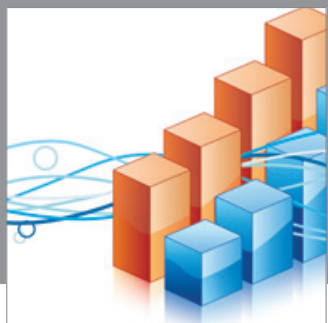

Advances in

Operations Research

mansans

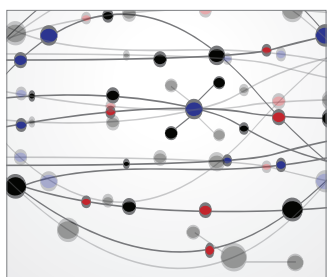

The Scientific World Journal
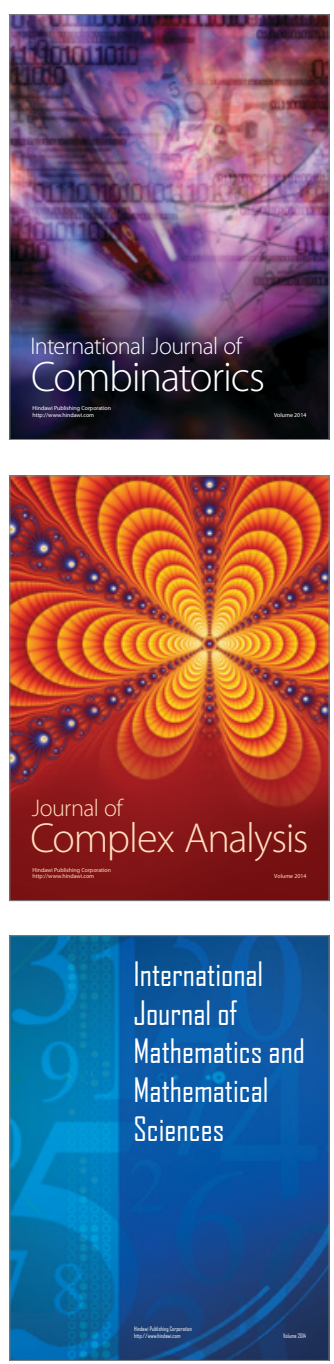
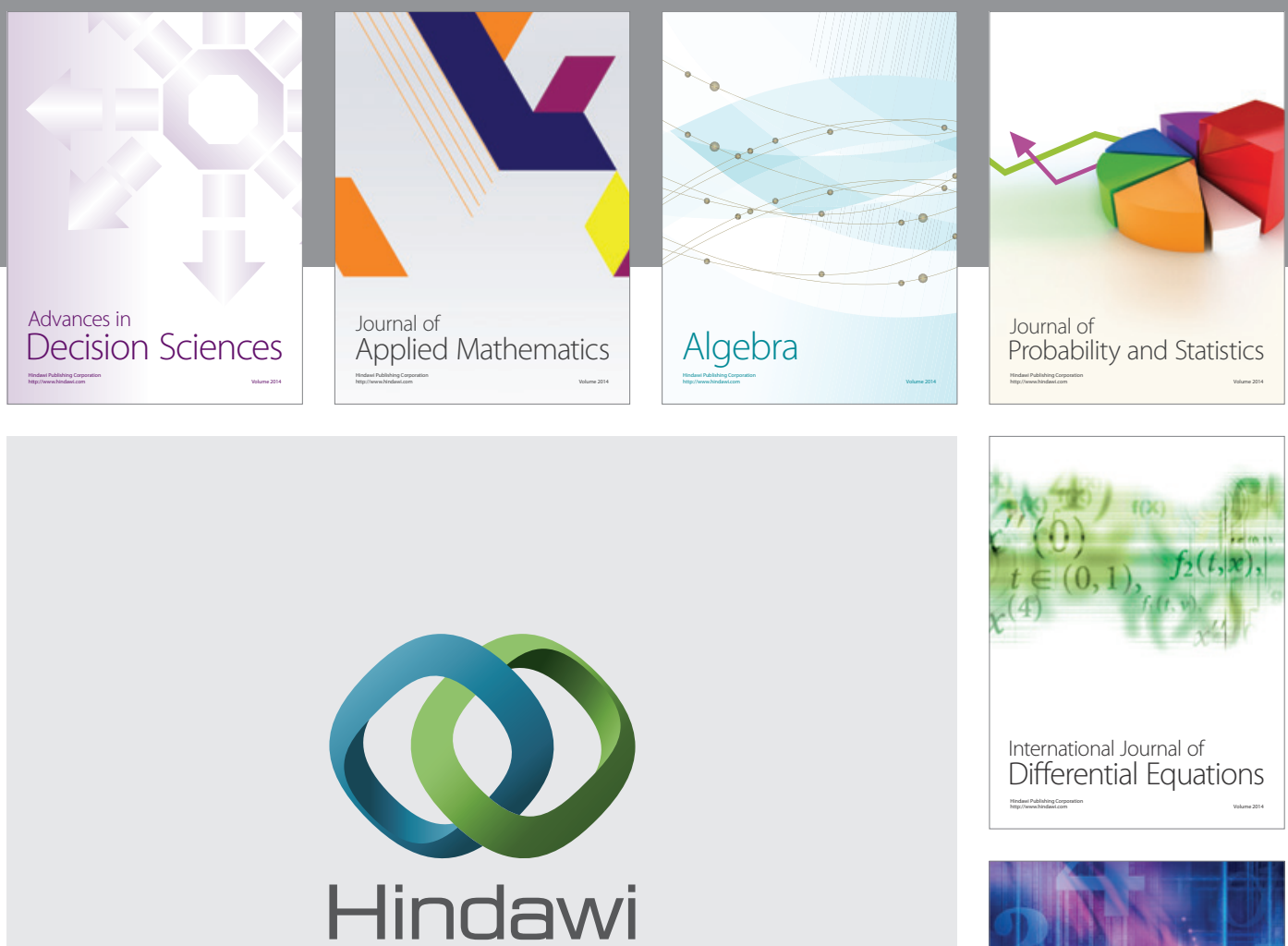

Submit your manuscripts at http://www.hindawi.com
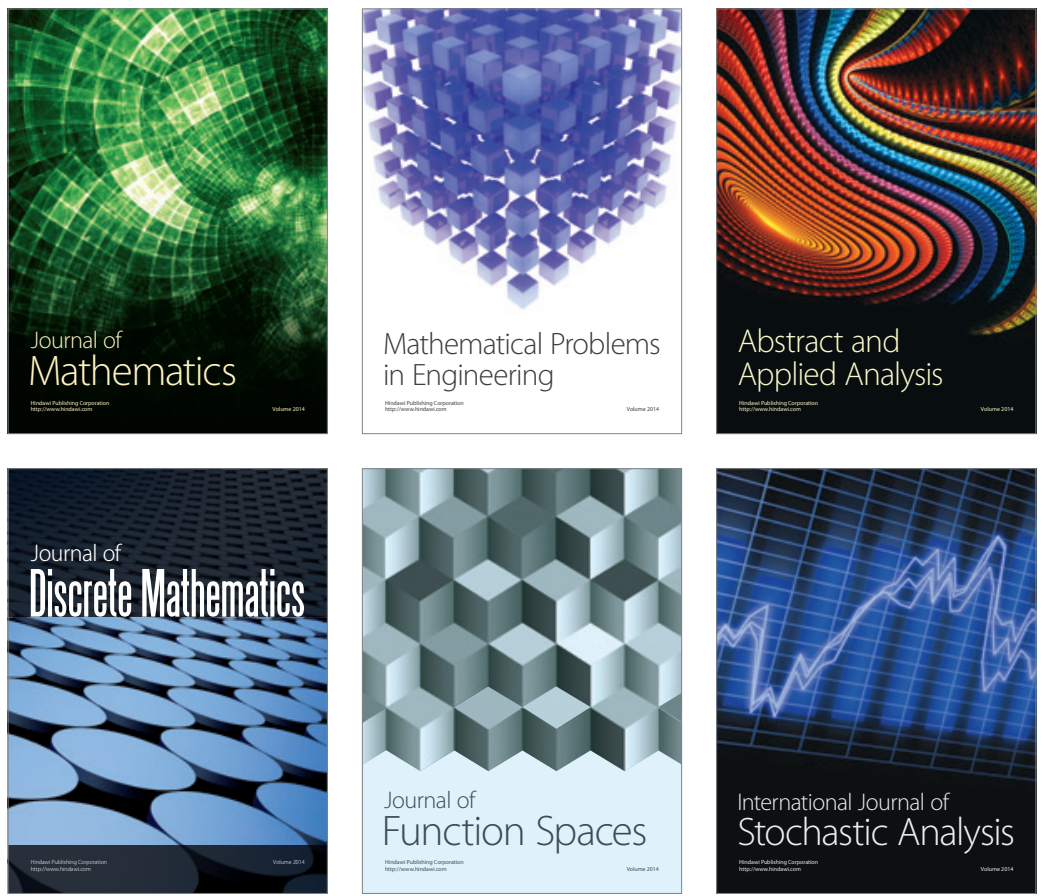

Journal of

Function Spaces

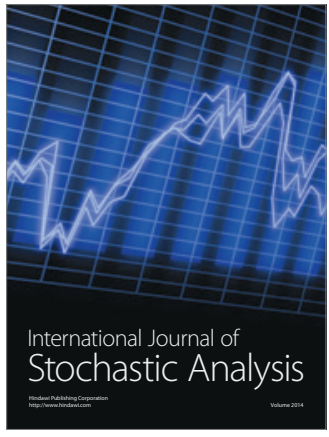

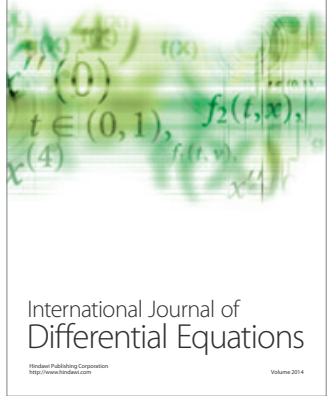
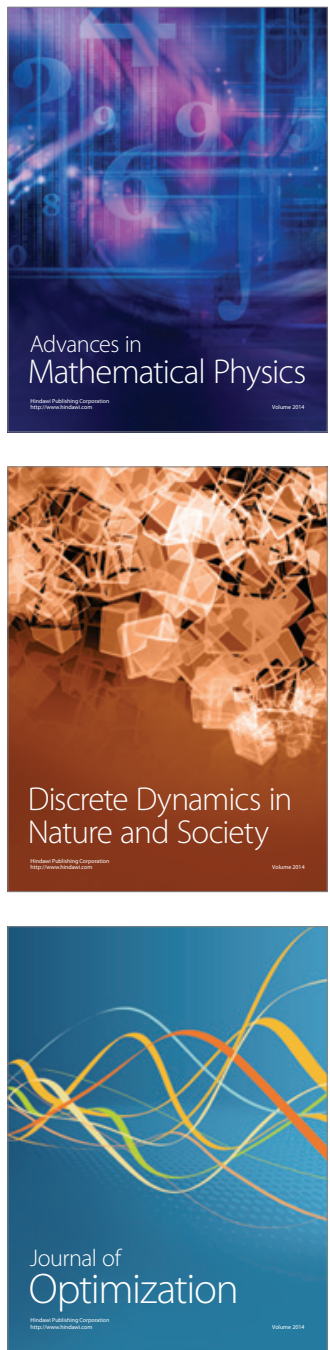